\title{
Thermal Mechanical Effects Research Based on Magnetic Thermal Mechanical Coupling Fields for Large Section Cables
}

\author{
Chunning Wang ${ }^{1}$, Mingxing $\mathrm{Fu}^{2}{ }^{2,}$, Fujue Wang ${ }^{3}$, Qiang $\mathrm{Chu}^{1}$, Wei Chen ${ }^{1}$ and Hongzhong $\mathrm{Ma}^{3}$ \\ ${ }^{1}$ Nanjing Power Supply Company of State Grid Jiangsu Electric Power Company, Nanjing 210008, China \\ ${ }^{2}$ Yancheng Power Supply Company of State Grid Jiangsu Electric Power Company, Yancheng 224002, China \\ ${ }^{3}$ College of Energy and Electrical Engineering, Hohai University, Nanjing 211100, China \\ ${ }^{*}$ Corresponding author
}

\begin{abstract}
In order to realize the quantitative calculation of large section cable's thermal stress and thermal strain, the finite element calculation model of cable's magnetic thermal mechanical coupling field is established, which takes a large cross-section cable fixed with hoops at both ends as a modeling object. The electromagnetic losses of cable are calculated, which are loaded on the finite element model as the excitation to calculate thermal stress and thermal strain of large section cable. Results show that thermal stress of large section cable is mainly distributed on the conductor and the metal sheath, but thermal stress of the other layers of cable is smaller. The relationship expression between thermal stress of the conductor and the metal sheath and load current is proposed by the method of data fitting, which provides a theoretical reference for formulating the scientific operation strategy of large section cable.
\end{abstract}

Keywords-large section cables; temperature field; finite element method; thermal mechanical effects; data fitting

\section{INTRODUCTION}

As an important carrier of power transmission, high voltage cables have been widely used in urban transmission and distribution system [1]. It is of great significance to improve the reliability of power cable operation to ensure the production and life of the city [2]. Due to the upgrading of urban power grid and the development needs of different customers, the demand of large section cables is increasing[3], [4]. Similar to other electrical equipments in power grids, some latent faults of power cables appear during operation, which is affected by the changes of external environment and power operation conditions. According to the statistics of the relevant power sector, the rate of power cables' faults caused by the thermal mechanical force in Nanjing is up to 36.2 percent in recent years [5], [6]. However, due to the lack of researches on the thermal machanical force at home and abroad, it is easy to ignore the influence of the thermal mechanical force on cables in the analysis of high voltage cable faults, then it fails to deal with the latent faults in the cable operation in time. Therefore, the research on the modeling of the thermal mechanical effects of large section cable can effectively evaluate the operating state of cable, and provide a theoretical reference for formulating the scientific strategy of the cable operation and maintenance.
Taking $220 \mathrm{kV}$ large section cable as the object, the magnetic thermal mechanical coupling model on large section cable for calculating thermal stress and thermal strain is established based on the finite element method. Then, the numerical and distribution characteristics of thermal stress and thermal stain of cables under different load currents are calculated on the basis of the simulation models. The relationship expression between thermal stress of the conductor and the metal sheath and load current is proposed by the method of data fitting.

\section{MATHEMATIC MOdEL ON CABLE'S COUPLiNG FiELDS}

The operation process of high voltage cables is essentially the interaction of cable's electromagnetic field, temperature field and stress field. To calculate thermal stress and thermal strain of cable, it needs to study mathematical models of cable's multi-physical fields. Taking the three-dimensional model of cable under air as the research object, mathematical models of cable's electromagnetic field, temperature field and mechanical field are put forward.

\section{A. Mathematical Model of Electromagnetic Field}

The vector magnetic potential control equation of each layer of cable can be expressed as follows:

$$
\nabla^{2} A=-\mu J_{\mathrm{c}}
$$

Where, $A$ is the vector magnetic pontential of the corresponding region. $\mu$ is the magnetic permeability of the material. represents the current density. For active regions such as the conductor and metal sheath of cable, $J_{\mathrm{c}}$ is not zero. For passive regions such as cable's insulation, outer sheath, and so on, $J_{\mathrm{c}}$ is directly taken as zero.

\section{B. Mathematical Model of Temperature Field}

The mathematical model of the three-dimensional temperature field of cable can be described as follows: 


$$
\rho c_{\mathrm{p}} \frac{\partial T}{\partial t}=\lambda\left(\frac{\partial^{2} T}{\partial x^{2}}+\frac{\partial^{2} T}{\partial y^{2}}+\frac{\partial^{2} T}{\partial z^{2}}\right)+q_{\mathrm{v}}
$$

Where, $T$ repersents the temperature $(\mathrm{K})$ at any point $(x, y, z)$ in the calculation region. $\lambda$ is the thermal conductivity of the materital $(\mathrm{W} /(\mathrm{m} \cdot \mathrm{K})) . \quad q_{\mathrm{v}}$ represents the unit volume heat production rate $\left(\mathrm{W} / \mathrm{m}^{3}\right) . \rho$ is the material density $\left(\mathrm{kg} / \mathrm{m}^{3}\right) . c_{\mathrm{p}}$ is the specific heat capacity of the material $(\mathrm{J} /(\mathrm{kg} \cdot \mathrm{K}))$. For regions containing heat sources, such as conductor and metal sheath, $q_{\mathrm{v}}$ is not zero. But for regions not containing heat sources, such as buffer layer and outer sheath, $q_{\mathrm{v}}$ is directly taken as zero.

\section{Mathematical Model of Mechanical Field}

The thermal stress of cable generates internally due to cable's temperature change. Its mathematical expression can be described as follows:

$$
\begin{cases}\sigma_{x}=H D_{\mathrm{v}}+2 G \varepsilon_{x}-2 G \alpha \Delta T, & \delta_{x y}=G \gamma_{x y} \\ \sigma_{y}=H D_{\mathrm{v}}+2 G \varepsilon_{y}-2 G \alpha \Delta T, & \delta_{y z}=G \gamma_{y z} \\ \sigma_{z}=H D_{\mathrm{v}}+2 G \varepsilon_{z}-2 G \alpha \Delta T, & \delta_{z x}=G \gamma_{z x}\end{cases}
$$

Where, $\sigma_{x}, \sigma_{y}, \sigma_{z}, \delta_{x y}, \delta_{y z}, \delta_{z x}$ represent stress components. $H$, $G$ represent lame constant. $\alpha$ is thermal expansion coefficient. $\Delta T$ is temperature difference. $\varepsilon_{x}, \varepsilon_{y}, \varepsilon_{z}, \gamma_{x y}, \gamma_{y z}, \gamma_{z x}$ represent strain components. $D_{\mathrm{v}}$ is volume strain, that $D_{\mathrm{v}}=\varepsilon_{x}+\varepsilon_{y}+\varepsilon_{z}$.

The strain components are described by the geometric equation as follows:

$$
\left\{\begin{array}{l}
\varepsilon_{x}=\frac{\partial u}{\partial x}, \quad \varepsilon_{y}=\frac{\partial v}{\partial y}, \varepsilon_{z}=\frac{\partial w}{\partial z} \\
\gamma_{x y}=\frac{\partial v}{\partial x}+\frac{\partial u}{\partial y}, \quad \gamma_{y z}=\frac{\partial w}{\partial y}+\frac{\partial v}{\partial z}, \gamma_{z x}=\frac{\partial u}{\partial z}+\frac{\partial w}{\partial x}
\end{array}\right.
$$

Where, $u, v, w$ represent the displacement components in three directions along $x, y$ and $z$ axis.

The stress equilibrium equations can be expressed as follows:

$$
\left\{\begin{array}{l}
\frac{\partial \sigma_{x}}{\partial x}+\frac{\partial \delta_{y x}}{\partial y}+\frac{\partial \delta_{z x}}{\partial z}+f_{x}=0 \\
\frac{\partial \sigma_{y}}{\partial y}+\frac{\partial \delta_{z y}}{\partial z}+\frac{\partial \delta_{x y}}{\partial x}+f_{y}=0 \\
\frac{\partial \sigma_{z}}{\partial z}+\frac{\partial \delta_{x z}}{\partial x}+\frac{\partial \delta_{y z}}{\partial y}+f_{z}=0
\end{array}\right.
$$

Where, $f_{x}, f_{y}, f_{z}$ represent the force components of cable in three directions along $x, y$ and $z$ axis.
Finally, the control equations of the stress field expressed by displacement components are obtained as follows:

$$
\left\{\begin{array}{l}
(H+G) \frac{\partial D_{\mathrm{v}}}{\partial x}+G \nabla^{2} u-2 G \alpha \frac{\partial \Delta T}{\partial x}+f_{x}=0 \\
(H+G) \frac{\partial D_{\mathrm{v}}}{\partial y}+G \nabla^{2} v-2 G \alpha \frac{\partial \Delta T}{\partial y}+f_{y}=0 \\
(H+G) \frac{\partial D_{\mathrm{v}}}{\partial z}+G \nabla^{2} w-2 G \alpha \frac{\partial \Delta T}{\partial z}+f_{z}=0
\end{array}\right.
$$

Where, while the cable is fixed along the direction of $x$ axis, the corresponding $u$ is taken as zero.

\section{Finite Element Model of LARGe Section CABle}

\section{A. Physical Model of Cable}

Taking $220 \mathrm{kV}$ cable with large section as the modeling object, which is fixed with hoops at both ends, the threedimensional model of magnetic thermal mechanical coupling fields on large section cable is established to calculate thermal stress and thermal strain of cable in operation. The structure parameters of $220 \mathrm{kV}$ cable are shown in Table I. When modeling, the length of cable is three meter. The physical model of cable is shown in Figure I.

\section{TABLE I. STRUCTURE PARAMETERS OF CABLE UNDER 220KV}

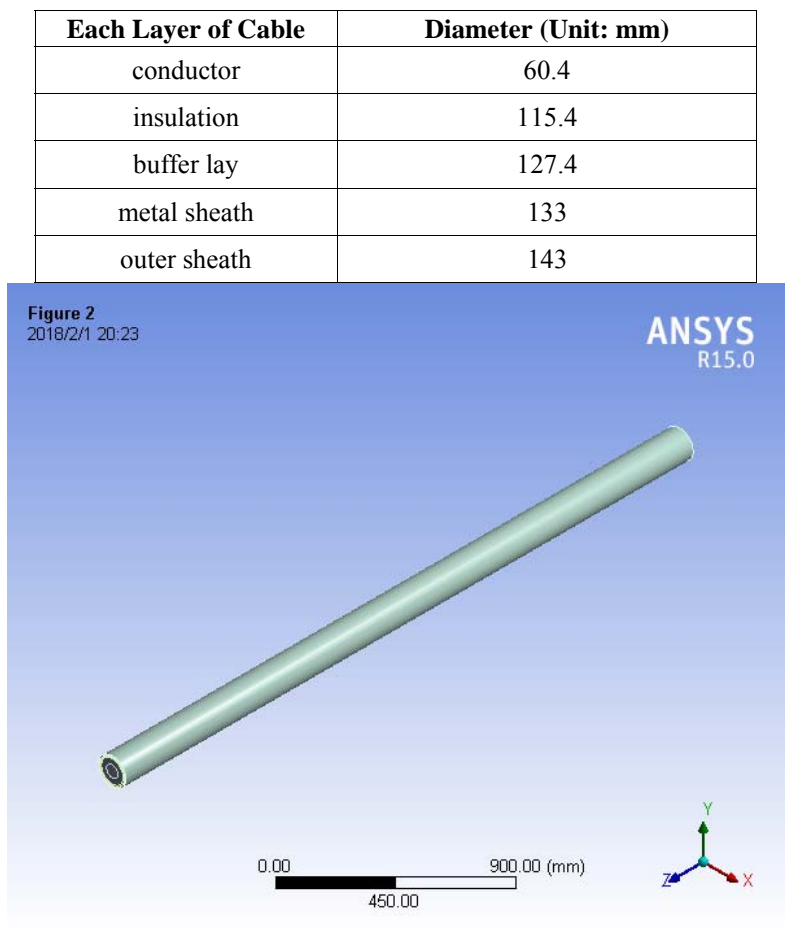

FIGURE I. PHYSICAL MODEL OF CABLE 


\section{B. Boundary Condition}

Considering the rapid attenuation of the vector magnetic potential in the air field out of the cable, the air field is closed to the $1000 \mathrm{~mm}$ of the cable body. The cross section of cable is set in the XY plane. The axis direction of cable is set along the $\mathrm{Z}$ axis. The solution domain model is shown in Figure II.

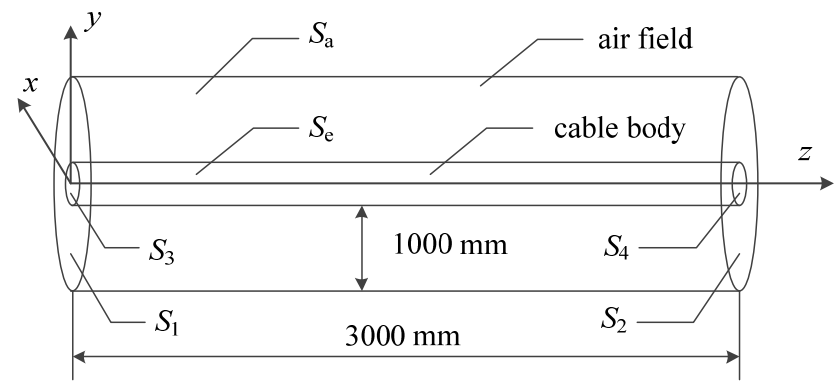

FIGURE II. A DIAGRAM OF THE CABLE SOLUTION DOMAIN MODEL

According to the schematic diagram of the solution domain model, the boundary conditions are set as follows.

\section{1) The boundary condition of electrimagnetic field:}

The vector magnetic potential has been rapidly reduced to zero at the air domain which is at a distance of $1000 \mathrm{~mm}$ from the cable body. Therefore, the boundart condition of the outer solution domain can be described as follow:

$$
\left.A\right|_{S_{\mathrm{a}}}=0
$$

The radial section of the two ends of the cable is magnetically insulated boundary condition. It can be described as follows:

$$
\left.\overrightarrow{n \times A}\right|_{S_{1}, S_{2}}=0
$$

Where, $\vec{n}$ represents the boundary normal vector.

2) The boundary condition of temperature field:

The cable under air mainly transfers heat to the outside in two ways, namely convention heat transfer and radition heat transfer. The boundary condition of convection heat transfer can be described as follows:

$$
-\left.\lambda \frac{\partial T}{\partial n}\right|_{S_{\mathrm{e}}}=\alpha\left(T_{\mathrm{e}}-T_{\mathrm{f}}\right)
$$

Where, $\alpha$ is the convection heat transfer coefficient of the outer surface of cable. $T_{\mathrm{e}}$ is the outer surface temperature of cable. $T_{\mathrm{f}}$ represents the ambient temperature.
The boundary condition of radiative heat transfer on cable surface can be described as follows:

$$
-\left.\lambda \frac{\partial T}{\partial n}\right|_{S_{\mathrm{e}}}=\sigma_{0} \varepsilon\left(T_{\mathrm{e}}^{4}-T_{\mathrm{f}}^{4}\right)
$$

Where, $\sigma_{0}$ represents the Stefan-Boltzmann constant. $\varepsilon$ represents the surface emissivity.

Considering that the axial temperature variation of cables is small enough to be neglected, the normal temperature gradient of the radial section at both ends of cable can be zero. It can be described as follows:

$$
-\left.\lambda \frac{\partial T}{\partial n}\right|_{S_{3}, S_{4}}=0
$$

\section{3) The boundary condition of mechanical field:}

Because of the pretightening force of the hoop on the cable, the axial displacement of the two ends of the cable is almost zero. It can be described as follows:

$$
\left.\vec{n} \cdot u\right|_{S_{3}, S_{4}}=0
$$

Where, $u$ is the component of the cable displacement. There is no external force and displacement constraint on the outer surface of the cable, namely $S_{\mathrm{e}}$.

\section{ANALySIS OF SimUlation RESUlts}

When current of $2280 \mathrm{~A}$ enters the conductor of cable, The radial section distribution of the electromagnetic losses of each layer in cable is shown in Figure III.

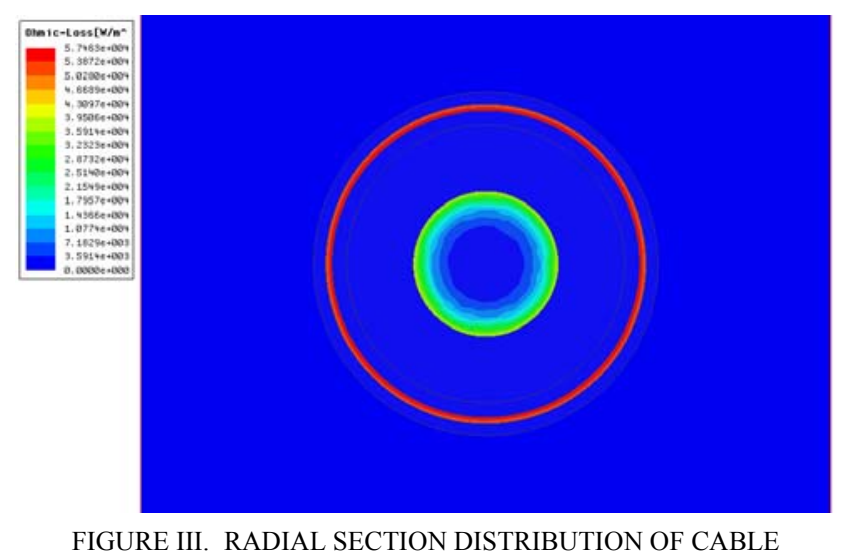

FIGURE III. RADIAL SECTION DISTRIBUTION OF CABLE ELECTRIMAGNETIC LOSSES.

The calculation results of the cable electromagnetic losses as thermal loads are coupled to the model of cable's temperature field. The radial section and the axial section of 
temperature field of each layer of cable are obtained shown in Figure IV. When the conductor current is $2280 \mathrm{~A}$, the temperature of conductor is $89.96{ }^{\circ} \mathrm{C}$. The temperature distribution of each layer of cable varies mainly in radial direction, and the temperature of cable axis does not change, that is, the temperature gradient of cable axis is basically zero.

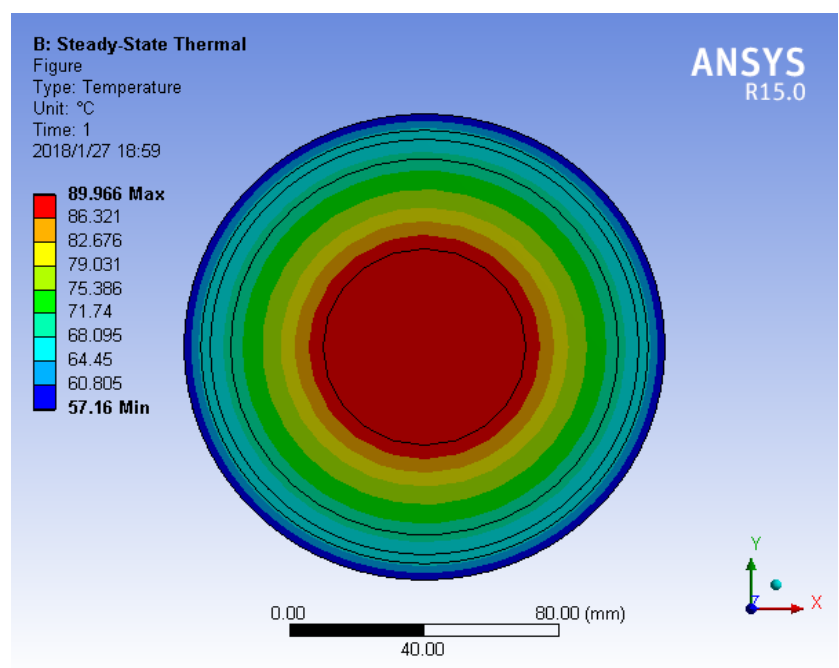

(A) RADIAL SECTION

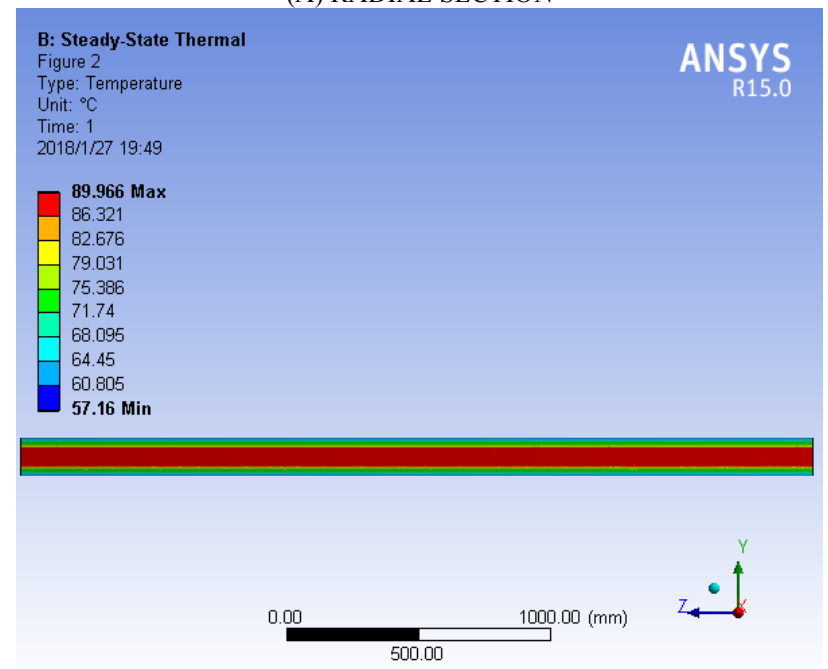

(B) AXIAL SECTION

FIGURE IV. DISTRIBUTION OF TEMPERATURE FIELD ON EACH LAYER OF CABLE.

In the same way, the radial and axial distribution of thermal stress and thermal strain of each layer of cable can be obtained by coupling the calculation results of temperature field on the calculation model of the stress field of cable, which is shown in Figure V and Figure VI.

Because the axial temperature gradient of the cable is basically zero, the heat of cable is transferred along the radial direction. Therefore, the thermal stress and the thermal strain of cable are basically unchanged along the axial direction, which mainly changes along the radial direction. From Figure V(A), it is known that the thermal stress of the running cable mainly distributes in the conductor and metal sheath of cable, and the values of thermal stress in other layers of cable are relatively small. When the temperature of cable conductor reaches $89.96{ }^{\circ} \mathrm{C}$, the value of thermal stress in cable conductor is 129.55 MPa, the value of thermal stress in metal sheath is $111.48 \mathrm{MPa}$ and the value of thermal stress in the other layers of cable is within $2 \mathrm{MPa}$. From Figure VI(A), it is known that the smaller value of thermal strain in the operating cable appears in the conductor and metal sheath of cable. Because the thermal expansion coefficient of metal material applied in conductor and metal sheath is smaller than that of the other layers.

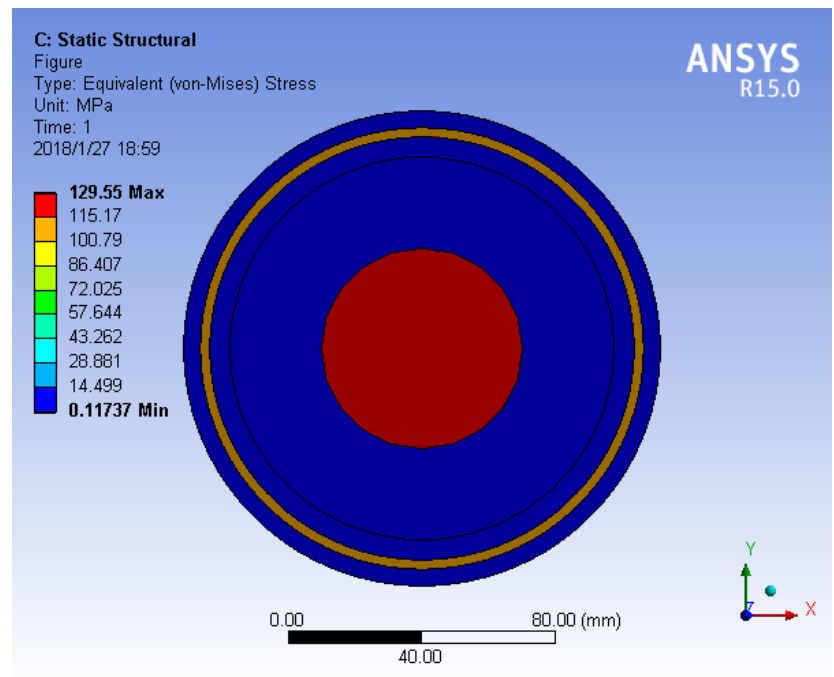

(A) RADIAL SECTION

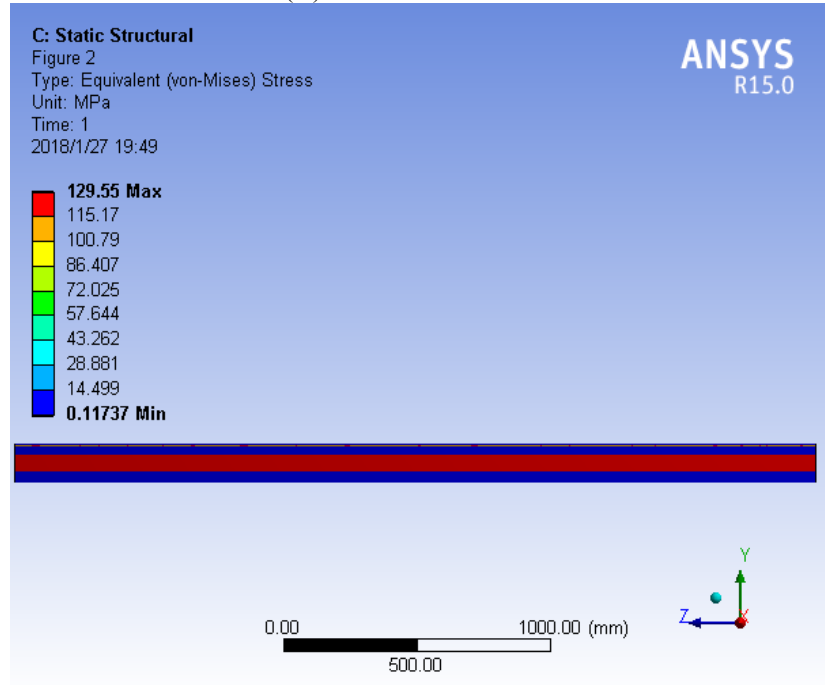

(B) AXIAL SECTION

FIGURE V. DISTRIBUTION OF THERMAL STRESS ON EACH LAYER OF CABLE. 


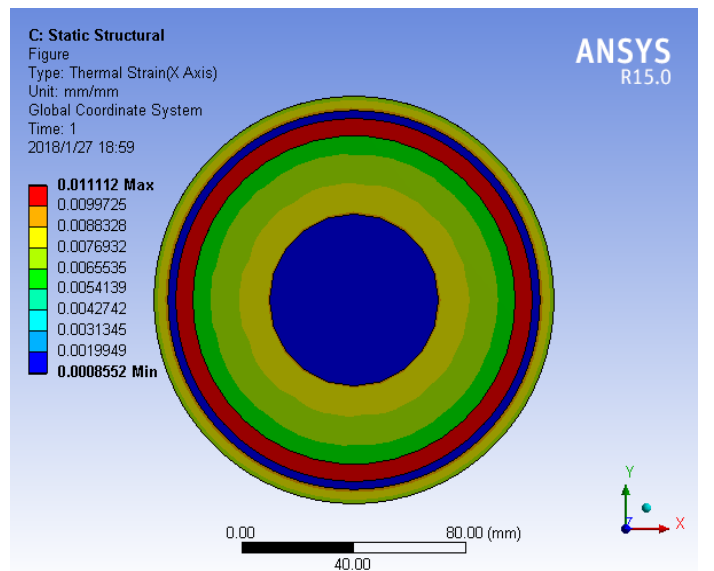

(A) RADIAL SECTION

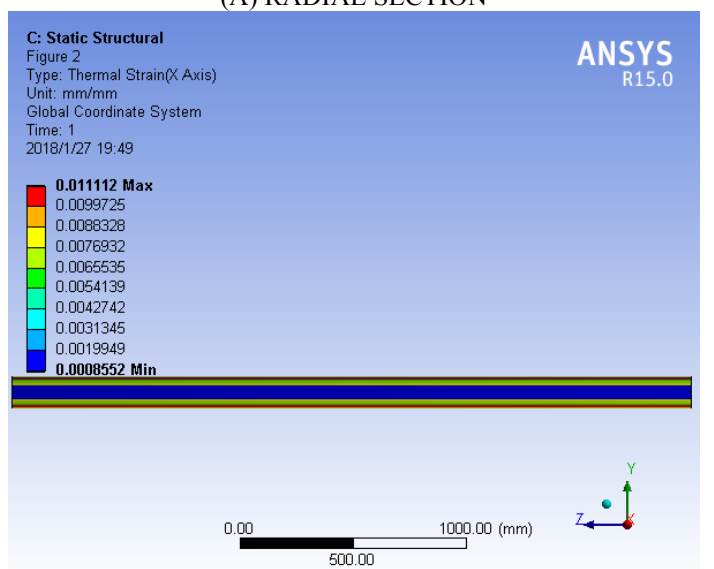

(B) AXIAL SECTION

FIGURE VI. DISTRIBUTION OF THERMAL STRAIN ON EACH LAYER OF CABLE.

By the way of changing the input value of current in cable conductor, the distribution results of thermal stress and thermal strain of each layer in cable are obtained under different load currents, which is shown in Figure VII and Figure VIII. According to the simulation datas of thermal stress of cable conductor and metal sheath under different load currents, the curves of relationship between the thermal stress and load current of conductor and metal sheath can be obtained, which is shown in figure IX. By the method of data fitting, the formula is obtained which expresses the relationship between thermal stress and load current of conductor and metal sheath, as follows:

$$
\begin{aligned}
& \sigma_{\mathrm{c}}=2.663 \times 10^{-5} I^{2}-1.396 \times 10^{-3} I+0.9439 \\
& \sigma_{\mathrm{m}}=2.27 \times 10^{-5} I^{2}-2.936 \times 10^{-4} I+0.5688
\end{aligned}
$$

Where, $\sigma_{\mathrm{c}}$ represents the thermal stress of cable conductor. (unit: MPa) $\sigma_{\mathrm{m}}$ represents the thermal stress of metal sheath of cable. (unit: Mpa) I is the load current of cable. (unit: A)

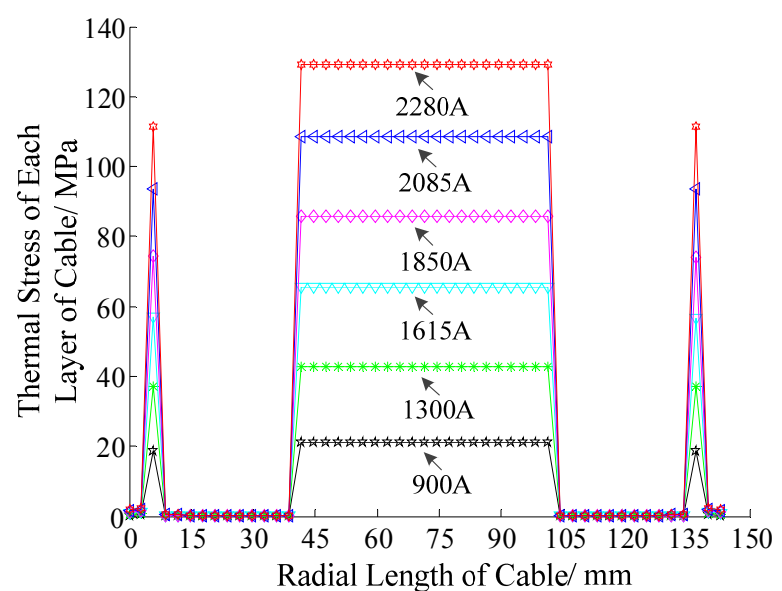

FIGURE VII. THERMAL STRESS OF EACH LAYER OF CABLE UNDER DIFFERENT CURRENTS.

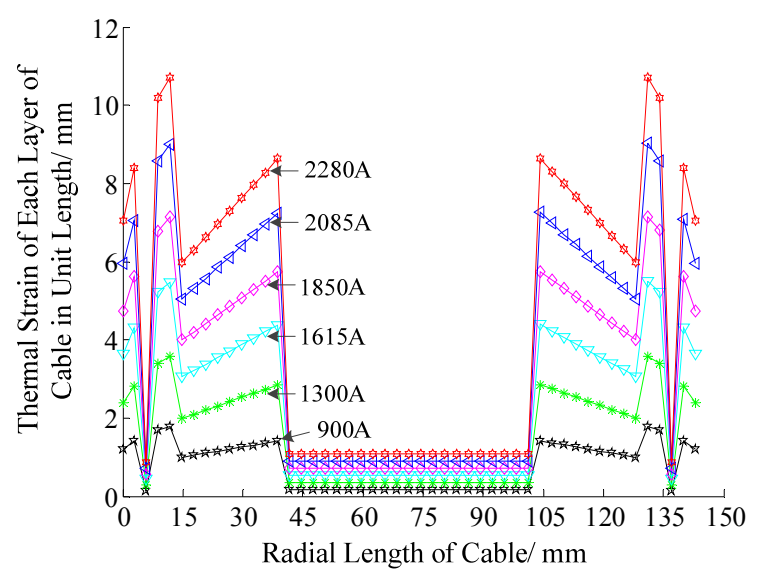

FIGURE VIII. THERMAL STRAIN OF EACH LAYER OF CABLE UNDER DIFFERENT CURRENTS.

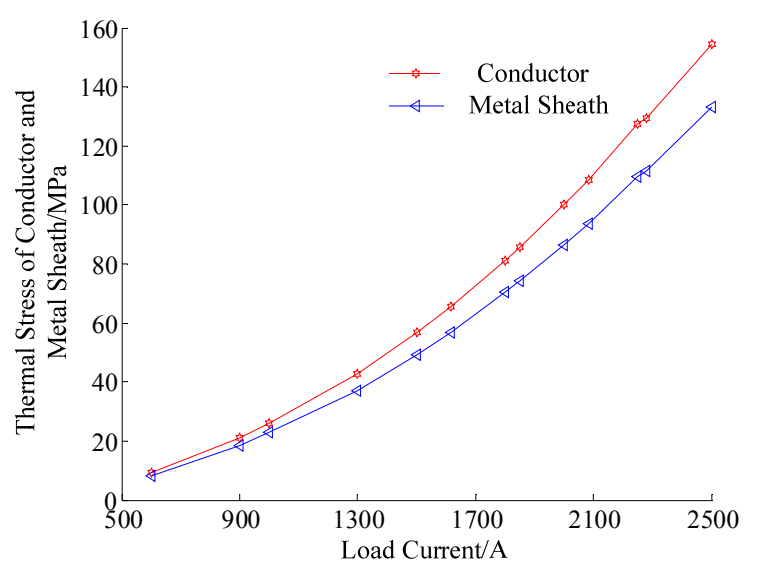

FIGURE IX. THE RELATION CURVE BETWEEN THERMAL STRESS OF CABLE CONDUCTOR AND METAL SHEATH AND LOAD CURRENT.

\section{CONCLUSION}

On the basis of the finite element method, the calculation model of magnetic thermal mechanical coupling fields on large 
section cables is put forward in this paper. According to simulation results, conclusions can be obtained as follows:

1) The temperature field of large section cable is mainly distributed along the radial direction, and the axial temperature gradient of it is zero.

2) The thermal stress of large section cable in operation is mainly distributed in conductor and metal sheath, but their thermal strain is very small.

3) The expression of quadratic function is obtained by the method of data fitting, which describes the relationship between thermal stress and load current of cable conductor and metal sheath.

\section{ACKNOWLEDGMENT}

This work is supported by Jiangsu Electric Power Company Key Science and Technology Project under Grant J2017070.

\section{REFERENCES}

[1] Marc D A, Francisco D L, "Adpative Soil Model for Real-time Thermal Rating of Underground Power Cables," IET Science, Measurement \& Technology. vol. 9, no. 6, pp. 654-660, 2015.

[2] ZHENG Yanling, "Research on Optimal Numerical Calcultion Model of Cable Groups Ampacity in Cable Group," High Voltage Engineering, Vol. 41, no. 11, pp. 3760-3765, 2015.

[3] LIU Gang, LEI Chenghua, LIU Yigang, "Analysis on Transient Error of Simplified Thermal Circuit Model for Calculating Conductor Temperature by Cable Surface Temperature," Power System Technology, Vol. 35, no. 4, pp. 212-217, 2011.

[4] LIANG Yongchun, "Technological Development in Evaluating the Temperature and Ampacity of Power Cables," High Voltage Engineering, Vol. 42, no. 4, pp. 1142-1150, 2016.

[5] WANG Jian, CHEN Chao, LI Qingmin, LIU Sihua, WANG Zhiyuan, "Thermal-induced Flexible Property of Gas Insulated Lines and Influenncing Factors Based on Thermal Mechanical Coupling Analysis," High Voltage Engineering, Vol. 43, no. 2, pp. 429-437, 2017.

[6] LIU Ji, ZHANG Mingze, ZHANG Zhenpeng, ZHAO Jiankang, CHEN Xin, "Influence of External Vibration on Laying Condition of $500 \mathrm{kV}$ XLPE Cables," High Voltage Engineering, Vol. 43, no. 2, pp. 673-681, 2017. 